\title{
Is a Level III Dissection Necessary for a Positive Sentinel Lymph Node in Melanoma?
}

\author{
JUKES P. NAMM, MD, ${ }^{1}$ ALFRED E. CHANG, MD, ${ }^{1}$ VINCENT M. CIMMINO, MD, ${ }^{1}$ RILEY S. REES, ${ }^{1}{ }^{1}{ }^{1}$ \\ TIMOTHY M. JOHNSON, MD, ${ }^{1,2}$ AND MICHAEL S. SABEL, MD ${ }^{1 *}$ \\ ${ }^{1}$ Department of Surgery, University of Michigan Health System, Ann Arbor, Michigan \\ ${ }^{2}$ Department of Dermatology, University of Michigan Health System, Ann Arbor, Michigan
}

\begin{abstract}
Background: For melanoma patients with a positive axillary SLN, the extent of ALND remains controversial, with debate over whether a level III dissection is needed.

Methods: We queried our IRB approved prospective database for patients with a positive axillary SLN who had a level I/II dissection only, and compared recurrence and complication rates to the existing literature.

Results: Between 1998 and 2008, 270 patients had 285 level I/II ALNDs for a positive SLN. Median number of SLN removed was 2, while the median number of involved SLN was 1 (range 1-4). An average of 18.7 nodes/ALND were removed, with 13\% having positive non-SLN. Post-operative complications occurred in 31 patients (11\%), primarily cellulitis (8\%). After a mean follow-up of 44 months, 14 patients had a regional recurrence in the axillary basin $(5 \%)$.

Conclusions: The complication rate and regional recurrence rate for patients undergoing a level I/II ALND for a positive SLN are either lower than or on par with reported series of ALND for level I, II, and III dissections, suggesting that in this setting, the level III dissection may be of minimal benefit.
\end{abstract}

J. Surg. Oncol. 2012;105:225-228. (C) 2011 Wiley Periodicals, Inc.

\section{KeY Words: melanoma; sentinel lymph node biopsy; axillary lymph node dissection}

\section{INTRODUCTION}

Sentinel lymph node (SLN) biopsy has proven to be safe, accurate, and highly prognostic for recurrence and survival in the setting of melanoma without clinical evidence of regional metastasis [1]. Patients who do not have evidence of metastasis in the SLN are spared a completion lymph node dissection (CLND) and the associated increased risk of complications, however for patients harboring disease in the SLN, a timely CLND of the draining lymph node basin is the standard recommendation.

For the axillary lymph nodes, which drain upper extremity and most upper trunk melanomas, the extent of that subsequent dissection is in debate. The guidelines established by the German Cancer Society recommend dissection of levels I-III for axillary CLND [2]. The group from John Wayne Cancer Institute also recommends dissection of all three levels in axillary CLND due to the variability of drainage patterns in second echelon nodes as well as the potential increased risk of recurrence with a lesser dissection [3]. Furthermore, the National Comprehensive Cancer Network guidelines state that for all positive SLN, an anatomically complete dissection of involved nodal basins is required [4]. However, there is still debate over whether a level III axillary dissection is necessary in these patients [5-7]. At our institution, we have been performing level I and II axillary CLND for most patients with positive SLN. We postulate that not only does this spare patients the morbidity associated with a level I-III dissection which can involve transection of the pectoralis minor at its insertion on the coracoid process and may be associated with an additional risk of lymphedema [8-12], but it may also achieve recurrence and survival rates on par with level I-III CLND reported in the literature.

\section{METHODS}

Utilizing our University of Michigan Institutional Review Board approved comprehensive, prospective melanoma database to identify a consecutive series of melanoma patients with positive axillary sentinel lymph nodes who underwent a subsequent complete dissection. We excluded patients under the age of 18 and patients who underwent ALND for clinically involved disease. Between January 1998 and December 2008, we identified 294 patients who underwent completion ALND for a positive SLN. Of these, 24 had levels 1-3 dissected while 270 underwent level 1 and 2 dissections. This latter group constitutes our final sample size for this study. Distant disease free survival time was determined as the time from wide local excision and SLN biopsy to the patient's last visit with no evidence of metastatic disease. Overall survival time was determined using a combination of hospital records and the Social Security Death Index (SSDI) and defined as the time from initial pathologic diagnosis to death or last visit.

Our SLN technique has been previously published [13]. Patients undergo same-day preoperative lymphoscinitigraphy in which 99mTc sulfur colloid (CIS-US, Inc., Bedford, MA) is injected intradermally around the primary lesion or biopsy site $2-4 \mathrm{hr}$ prior to surgery to identify lymphatic basins and in-transit nodes at risk. After induction of anesthesia, either isosulfan blue dye or diluted methylene blue dye is injected intradermally around the primary lesion or biopsy site and the area is massaged to promote lymphatic flow. After approximately 5-10 min, a handheld gamma probe (Navigator GPS; US Surgical, Cincinnati, OH) is used to identify the area of maximal counts over the lymphatic basins identified by preoperative lymphoscintigraphy. A small incision is made directly overlying the

Grant sponsor: NIH Institutional Training Grant; Grant number: T32.

*Correspondence to: Michael S. Sabel, MD, 3304 Cancer Center, 1500 East Medical Center Drive, Ann Arbor, MI 48109. Fax: +1-734-6479647. E-mail: msabel@umich.edu

Received 11 January 2011; Accepted 28 July 2011

DOI 10.1002/jso.22076

Published online 22 August 2011 in Wiley Online Library (wileyonlinelibrary.com). 
hot spot and carried through the subcutaneous tissue in to the nodebearing fat. Any blue, hot (defined as the hottest node and any other nodes greater than $10 \%$ of the counts per minute of the hottest node) or palpably suspicious lymph nodes are considered SLNs. SLN specimens were fixed in $10 \%$ neutral-buffered formalin, serially sectioned longitudinally at 2-3 mm intervals, and entirely embedded in paraffin. Hematoxylin and eosin (H\&E) staining was performed on two 5 - $\mu$ m-thick sections from each tissue block. In addition, four unstained serial sections were prepared for possible future immunohistochemical analysis. If SLN(s) were negative by routine light microscopy, immunohistochemical staining was routinedly performed using antibodies to the melanoma markers Melan-A (1:12.5 dilution; DAKO Corporation, Carpinteria, CA) and S-100 protein (1:500 dilution; DAKO Corporation). Tumor burden of the positive SLNs (i.e., percentage of surface area involved) was measured using our previously described technique [14]. Nonsentinel nodes in the axillary lymph node dissection (ALND) specimen were evaluated by routine bivalving and $\mathrm{H} \& \mathrm{E}$ staining.

Level I and II axillary lymph node dissections were defined as mobilization and retraction of the pectoralis major (to include the interpectoral nodes) and pectoralis minor to include the level II lymph nodes. As previously stated, patients who underwent division of the pectoralis minor muscle to gain access to the level III nodes, or excision of the level III nodes under the pectoralis major but medial to the pectoralis minor, were excluded from review.

\section{RESULTS}

Over a 10-year period from 1998 to 2008,270 patients with melanoma metastatic to an axillary sentinel lymph node underwent a subsequent level I and II axillary dissection. This patient population consisted of 110 women (41\%) and 160 men (59\%). The median age was 50, with a range from 19 to 93 years. Only two patients (1\%) had primary melanomas on the head and neck, while 201 (74\%) had trunk melanomas and 67 (25\%) had melanomas of the upper extremity. Patient and tumor characteristics of the cohort are shown in Table I.

Of the 270 patients, 51 patients (19\%) had drainage to more than one basin, with 38 patients mapping to bilateral axillae, 7 patients mapping to both the axilla and groin, 4 mapping to the axilla and neck and 2 patients mapping to epitrochlear nodes in addition to axillary nodes. Fifteen of the patients underwent bilateral ALND (for a total of 285 ALND's in 270 patients). The median number of SLN removed was 2 (average 2.8) with a range of 1-20. The median number of involved SLN was 1 (average 1.8) with a range of 1-4. Extranodal extension was identified in 21 (8\%), absent in $244(90 \%)$, and unknown for 5 cases (2\%). On the subsequent node dissection, there was an average of 18.7 nodes in the specimen, ranging from 2 to 50. Of the 285 ALNDs performed, positive non-sentinel lymph nodes were found in $37(13 \%)$. The mean number of positive nodes in the ALND specimen was 0.42 , ranging from 0 to 38. Postoperative complications occurred in 31 patients (11\%). Infectious complications, primarily cellulitis requiring antibiotics, occurred in 22 patients $(8 \%)$. Seroma after drain removal occurred in 6 patients (2\%) and 2 patients (1\%) developed an axillary hematoma. One patient had a temporary winged scapula which resolved with physical therapy.

The median follow-up for this group was 44 months. There were six patients for whom no significant follow-up after surgery was available. Among the remaining 264 patients, 6 patients (2\%) suffered a local recurrence at the site of the primary melanoma and 19 patients $(7 \%)$ developed in-transit disease. There were 57 patients (22\%) who ultimately developed distant disease. Our primary endpoint however, was regional recurrence, and this was seen in 14 patients $(5 \%)$. Of these 14 patients, $10(71 \%)$ also had distant
TABLE I. Patient and Tumor Characteristics of the Study Cohort

\begin{tabular}{|c|c|}
\hline Average age (range) & $50(19-93)$ \\
\hline Gender (male/female) & $160(59 \%) / 110(41 \%)$ \\
\hline \multicolumn{2}{|l|}{ Site } \\
\hline $\mathrm{H} \& \mathrm{~N}$ & $2(1 \%)$ \\
\hline Trunk & $201(74 \%)$ \\
\hline Upper extremity & $67(25 \%)$ \\
\hline \multicolumn{2}{|l|}{ Histologic subtype } \\
\hline Superficial spreading & $132(49 \%)$ \\
\hline Nodular & $75(28 \%)$ \\
\hline Other/unclassified & $62(23 \%)$ \\
\hline Breslow thickness, median (range) & $2.2 \mathrm{~mm}(0.8-18)$ \\
\hline \multicolumn{2}{|l|}{ Ulceration } \\
\hline Yes & $96(36 \%)$ \\
\hline No & $165(61 \%)$ \\
\hline Unknown & $9(3 \%)$ \\
\hline Mitotic rate, mean (range) & $5.25(0-38)$ \\
\hline \multicolumn{2}{|l|}{ Satellitosis } \\
\hline Yes & $14(5 \%)$ \\
\hline No & $237(88 \%)$ \\
\hline Unknown & $19(7 \%)$ \\
\hline \multicolumn{2}{|l|}{ Angiolymphatic invasion } \\
\hline Yes & $38(14 \%)$ \\
\hline No & $213(79 \%)$ \\
\hline Unknown & $19(7 \%)$ \\
\hline \# SLN removed, mean (range) & $2.8(1-20)$ \\
\hline \# Pos SLN, mean (range) & $1.28(1-4)$ \\
\hline \# nodes in ALND, mean (range) & $18.68(4-50)$ \\
\hline$\%$ with positive NSLN & $37(13 \%)$ \\
\hline
\end{tabular}

SLN, sentinel lymph node; ALND, axillary lymph node dissection.

recurrences, $60 \%$ of which were synchronous to discovery of the regional recurrence. The remaining four patients developed distant disease more than 6 months after their regional recurrence was detected. Four patients have not yet recurred after resection of the regional recurrence. Four of the 14 axillary recurrences were nodules in the skin near the ALND incision, while only one was clearly documented as a level III recurrence. Among the patients who did have an axillary recurrence, only 2 of 14 (14\%) had positive nonsentinel nodes, compared with $12 \%$ of patients who did not recur. At the point of last follow-up, $191(71 \%)$ were alive, $180(67 \%)$ without disease, $9(3 \%)$ with disease and $2(1 \%)$ with an unknown disease status. Seventy-nine patients $(29 \%)$ had died, $52(19 \%)$ of metastatic melanoma, 19 (7\%) without melanoma, and 8 died of unknown reasons.

\section{DISCUSSION}

The purpose of this retrospective study was to review our institutional experience with level I/II ALND for patients with microscopic regional disease detected on SLN biopsy, with particular regards to regional control. We sought to compare our morbidity and recurrence data to published data for ALND since the advent of SLN for melanoma. Unfortunately this objective is limited by a paucity of contemporary data specifically looking at ALND following a positive SLN. The lion's share of the literature focuses on either ALND for clinically evident disease, or regional recurrence for nodal dissections overall, grouping together neck, axillary and inguinal dissections. In addition, many studies do not specifically document the extent of the axillary dissection.

In our series, postoperative complications occurred in $11 \%$ of patients, with infectious complications in $8 \%$. Wrightson et al. [15] reporting for the Sunbelt Melanoma Group, described a $19.9 \%$ complication rate among 262 patients undergoing ALND for a positive SLN. Although they did not specifically describe the extent of the 
ALND, most cases are thought to be level I-III dissections. Kretschmer et al. [16] reported a 35\% short-term complication rate ( $20.7 \%$ wound infection and $18.9 \%$ seroma) in a series of 70 level IIII dissections. de Vries et al. [17] reported similar results after level I-III CLND with a $14 \%$ wound infection rate and a $36 \%$ seroma rate.

Our primary endpoint of interest was the regional recurrence rate. With a median follow-up of 44 months, we report a regional recurrence rate of $5 \%$. This is very similar to a smaller series (48 patients) by Guggenheim et al. [18] examining level I and II dissections, including level III only when there were clinically suspicious nodes. With a median follow-up of 38.8 months, they reported a regional recurrence rate of $4.3 \%$. As a level III dissection was only performed in one patient, the authors concluded that level III dissections are not necessary for regional control.

We sought to compare these results to series where level III dissections were routinely performed, but there is a paucity of published data to compare with. Most studies reporting regional recurrence after CLND for a positive SLN grouped together all dissected basins. Gershenwald et al. [19] reported a series of 101 patients who underwent CLND with a 30-month median follow-up, finding a $10 \%$ regional recurrence rate. Clary et al. [20] reported a regional recurrence rate of $14 \%$ among 56 patients undergoing CLND for a positive SLN, with a median follow-up of 27 months. Leiter et al. [21] reported a $19.1 \%$ regional recurrence rate after CLND. In all three of these studies, it is not specified how many patients had axillary recurrences after ALND, nor the extent of the ALND performed. Veenstra et al. [22] report a $4 \%$ regional recurrence rate overall, and did specify that when an ALND was performed, levels I-III were included, however they did not tease out the axillary recurrence rate specifically.

Despite multiple references in the literature to the "controversy" of whether a level III dissection is necessary, there are very few published papers addressing this particular issue. In our series of level I and II only ALND's for a positive SLN, our regional recurrence rate is lower than, or on par with, comparable series. This suggests minimal benefit to the routine inclusion of level III nodes in this situation. However, it is difficult to define the adequacy of the ALND based on anatomic landmarks. In many prospective trials, the number of nodes within the axillary specimen is the benchmark of a successful dissection. Balch et al. [23] suggested that 10 or more nodes need to be retrieved in an adequate ALND. Morton et al. [24] in the MSLT-I trial recommended 15 or more nodes for an ALND ( $\geq 30$ for a neck dissection and $\geq 8$ for an ILND), and subsequently reported a $4.2 \%$ regional recurrence rate (again, not specifying axillary recurrence rates).

Spillane et al. [25] examined 863 ALND's performed by Sydney Melanoma Unit surgeons, who routinely perform level I-III dissections, and found that the mean number of nodes in the specimen was 21.9 , with at least 10 or more nodes in $90 \%$ of cases. This included both ALND's for a positive SLN and clinically evident axillary disease. In our series of patients with a positive SLN, where level III was not specifically addressed, our mean number of nodes was 18.7. Less than 10 nodes were retrieved in 28 cases, for a similar $90 \%$ rate of ALND's with 10 or more nodes. Likewise, the series by Guggenheim et al. [18] had a median of 18 nodes per level I and II dissection. These numbers surpass the median number of nodes found in some studies of level I-III ALND. Therefore an adequate ALND for a positive SLN may not necessarily have to incorporate the nodes medial to the pectoralis minor muscle, but should include meticulous clearance of nodes by landmarks for levels I and II.

A limitation to this study is the retrospective nature of this review. As this review incorporates the cases of six different surgeons, variability in technique cannot be accounted for. Complete or partial level III dissection can be accomplished in some cases without division of the pectoralis minor muscle, so that what one surgeon calls a level I and II dissection, another surgeon might consider a partial level III dissection. However, it must be emphasized that complete level II dissections were performed, which mandates ample mobilization of the pectoralis minor muscle. We find that the use of the Thompson axillary retractor helps considerably with this component. Careful examination of the specimen and identification of palpable or suspicious nodes (macroscopic disease) should also be employed so that more extensive dissection can be performed selectively. However, many patients have enlarged nodes after a prior SLN biopsy that are reactive and not malignant, therefore it may be reasonable to consider frozen section analysis prior to dividing the pectoralis minor if dissection of level III is being considered.

Other technical points bear mentioning as well, as there are some aspects to the ALND performed for melanoma that may not be addressed as strictly by surgeons used to performing an ALND for breast cancer. The melanoma surgeon must pay particular attention to the tissue lateral to the thoracodorsal bundle, where the lateral (axillary) group of nodes is located. These nodes receive most of the lymph draining from the upper extremity, so they rarely contain breast cancer metastases but are commonly the first group of nodes draining melanomas on the upper extremity. The thoracodorsal bundle should not be considered the lateral margin. Clearing the lymphatic tissue superior to the axillary vein is also important in preventing recurrence, particularly for melanomas located on the shoulder or lateral back. The posterior (subscapular) node group consists of 6 or 7 nodes that lie along the posterior wall of the axilla at the lateral border of the scapula, and receive drainage primarily from the posterior neck and trunk. Therefore the dissection must extend sufficiently inferior to clear the lowermost reaches of the axilla, and the nodal tissue must be cleared down to the level of the subscapularis muscle.

In conclusion, our large, single-institution, retrospective series of level I and II ALND performed for positive SLN among melanoma patients demonstrate a complication rate of $11 \%$ and a regional recurrence rate of $5 \%$. These results are either lower than or on par with reported series of ALND for level I, II and III dissections, suggesting that when ALND is performed for a positive SLN, the level III dissection may be of minimal benefit with added morbidity.

\section{REFERENCES}

1. Morton DL, Thompson JF, Cochran AJ, et al.: Sentinel-node biopsy or nodal observation in melanoma. N Engl J Med 2006; 355:1307-1317.

2. Garbe C, Hauschild A, Volkenandt M, et al.: Evidence-based and interdisciplinary consensus-based German guidelines: Systemic medical treatment of melanoma in the adjuvant and palliative setting. Melanoma Res 2008;18:152-160.

3. Essner R: Surgical treatment of malignant melanoma. Surg Clin N Am 2003;83:109-156.

4. NCCN: Clinical Practical Guidelines in Oncology- Melanoma. 2011. (Accessed 12/21, 2010, at http://www.nccn.org/profesionals/physician_gls/PDF/melanoma.pdf.).

5. Karakousis CP: Therapeutic node dissections in malignant melanoma. Ann Surg Oncol 1998;5:473-482.

6. Meyer T, Merkel S, Gohl J, et al.: Lymph node dissection for clinically evident lymph node metastases of malignant melanoma. Eur J Surg Oncol 2002;28:424-430.

7. Serpell JW, Carne PW, Bailey M: Radical lymph node dissection for melanoma. ANZ J Surg 2003;73:294-299.

8. Sakorafas GH, Peros G, Cataliotti L, et al.: Lymphedema following axillary lymph node dissection for breast cancer. Surg Oncol 2006;15:153-165.

9. Larson D, Weinstein M, Goldberg I, et al.: Edema of the arm as a function of the extent of axillary surgery in patients with stage 
I-II carcinoma of the breast treated with primary radiotherapy. Int J Radiat Oncol Biol Phys 1986;12:1575-1582.

10. Silberman H: Axillary lymphadenectomy for breast cancer: Impact on survival. In: Silberman H, Silberman A, editors. Surgical oncology: Multidisciplinary approach to difficult problems. London: Arnold; 2002. pp. 369-385.

11. Kiel K, Rademaker AW: Early-stage breast cancer: Arm edema after wide excision and breast irradiation. Radiology 1996;1998: 279-283.

12. Suneson BL: Clinical incidence of lymphedema in breast cancer patients in Jonkoping County, Sweden. Eur J Cancer Care 1996; 5:1-12.

13. Sondak VK, Taylor JM, Sabel MS, et al.: Mitotic rate and younger age are predictors of sentinel lymph node positivity: Lessons learned from the generation of a probabilistic model. Ann Surg Oncol 2004;11:247-258.

14. Frankel TL, Griffith KA, Lowe L, et al.: Do micromorphometric features of metastatic deposits within sentinel nodes predict nonsentinel lymph node involvement in melanoma? Ann Surg Oncol 2008;15:2403-2411.

15. Wrightson WR, Wong SL, Edwards MJ, et al.: Complications associated with sentinel lymph node biopsy for melanoma. Ann Surg Onc 2003;10:676-780.

16. Kretschmer L, Thoms KM, Peeters S, et al.: Postoperative morbidity of lymph node excision for cutaneous melanoma-sentinel lymphonodectomy versus complete regional lymph node dissection. Melanoma Res 2008;18:16-21.

17. de Vries M, Vonkeman WG, van Ginkel RJ, et al.: Morbidity after axillary sentinel lymph node biopsy in patients with cutaneous melanoma. Eur J Surg Oncol 2005;31:778-783.
18. Guggenheim MM, Hug U, Jung FJ, et al.: Morbidity and recurrence after completion lymph node dissection following sentinel lymph node biopsy in cutaneous malignant melanoma. Ann Surg 2008;247:687-693.

19. Gershenwald JE, Berman RS, Porter G, et al.: Regional nodal basin control is not compromised by previous sentinel lymph node biopsy in patients with melanoma. Ann Surg Oncol 2000; 7:226-231.

20. Clary BM, Brady MS, Lewis JJ, et al.: Sentinel lymph node biopsy in the management of patients with primary cutaneous melanoma: Review of a large single-institution experience with an emphasis on recurrence. Ann Surg 2001;233:250-258.

21. Leiter U, Buettner PG, Bohnenberger K, et al.: Sentinel lymph node dissection in primary melanoma reduces subsequent regional lymph node metastasis as well as distant metastasis after nodal involvement. Ann Surg Oncol 17:129-137.

22. Veenstra HJ, van der Ploeg IM, Wouters MW, et al.: Reevaluation of the locoregional recurrence rate in melanoma patients with a positive sentinel node compared to patients with palpable nodal involvement. Ann Surg Oncol 17:521-526.

23. Balch CM, Durant JR, Bartolucci AA: The impact of surgical quality control in multi-institutional group trials involving adjuvant cancer treatments. Ann Surg 1983;198:164-167.

24. Morton DL, Cochran AJ, Thompson JF, et al.: Sentinel node biopsy for early-stage melanoma: Accuracy and morbidity in MSLT-I, an international multicenter trial. Ann Surg 2005;242: 302-313.

25. Spillane AJ, Cheung BL, Stretch JR, et al.: Proposed quality standards for regional lymph node dissections in patients with melanoma. Ann Surg 2009;249:473-480. 